\title{
Distribution spatiale du singe à ventre rouge, Cercopithecus erythrogaster erythrogaster Gray et les menaces pesant sur sa conservation durable au Togo
}

\author{
K.G. Eric AGBESSI ${ }^{1 *}$, Moumouni OUEDRAOGO ${ }^{2}$, Mouhameth CAMARA ${ }^{3}$, \\ Hoinsoudé SEGNIAGBETO ${ }^{4}$, Mariano B. HOUNGBEDJI ${ }^{5}$ et André T. KABRE ${ }^{1}$ \\ ${ }^{1}$ Université Polytechnique de Bobo-Dioulasso. \\ ${ }^{2}$ Université de Ouagadougou. \\ ${ }^{3}$ Université de Thiès. \\ ${ }^{4}$ Université de Lomé. \\ ${ }^{5}$ Université d'Abomey-Calavi. \\ *Auteur correspondant ; E-mail: agbe18@gmail.com ; Tel: 0022890166674.
}

\section{RESUME}

Le singe à ventre rouge (Cercopithecus erythrogaster erythrogaster Gray) qui est une sous espèce en danger et endémique du Dahomey Gap est très peu documenté au Togo. Pour connaître sa distribution spatiale, les formes et l'ampleur des menaces auxquelles il est soumis dans le complexe d'aires protégées Togodo, des investigations et prospections pédestres ont été menées dans six zones correspondant aux six villages riverains et dans la forêt sacrée de Godjinmé située à $9 \mathrm{~km}$ environ au Sud du complexe. Les effectifs des singes identifiés ainsi que, leurs coordonnées géographiques, leurs activités au moment de l'observation, la distance d'observation et autres informations utiles sont portés sur une fiche technique. Les données collectées ont été traitées grâce à Ms Excel 2013, au logiciel R version 3.1.3. Le logiciel libre Qgis 2.10 a été exploité pour la représentation de la distribution de la sous espèce dans le complexe. Il a été dénombré, 99 individus dans le complexe et 5 dans la forêt sacrée de Godjinmé. Cependant, de nombreuses pressions menacent la survie des populations de ces primates non humains. On note le braconnage, la destruction et les perturbations de leurs habitats par l'agriculture, la transhumance et l'exploitation forestière.

(C) 2017 International Formulae Group. All rights reserved.

Mots clés: Cercopithecus erythrogaster erythrogaster, Dahomey Gap, Togodo, Godjinmé, effectifs, inventaire.

\section{Spatial distribution of red-bellied monkeys, Cercopithecus erythrogaster erythrogaster Gray and threats to its sustainable conservation in Togo}

\begin{abstract}
The red-bellied monkey (Cercopithecus erythrogaster erythrogaster Gray) which is a endangered and endemic sub-species of the Dahomey Gap is very poorly documented in Togo. In order to know its spatial distribution, the forms and the scale of the threats to which it is subjected in the Togodo protected area
\end{abstract}


complex, investigations and pedestrian surveys were carried out in six zones corresponding to the six bordering villages and in the sacred forest of Godjinmé located about $9 \mathrm{~km}$ in South of the complex. The numbers of monkeys identified and their geographical coordinates, activities at the time of observation, the viewing distance and other useful information are recorded on a data sheet. The collected data were processed using MS Excel 2013, the R version 3.1.3 software. Free software Qgis 2.10 was used for the representation of the distribution of the species in the protected area complex. In total, 99 individuals were counted in the complex and 5 in the sacred forest of Godjinmé. However, many pressures threaten the survival of populations of these non-human primates. Poaching, destruction and disturbance of their habitats by agriculture, transhumance and logging are noted.

(C) 2017 International Formulae Group. All rights reserved.

Keywords: Cercopithecus erythrogaster erythrogaster, Dahomey Gap, Togodo, Godjinme, population, inventory.

\section{INTRODUCTION}

La protection des zones forestières soumises à de fortes dégradations est une priorité de longue date. Dès l'époque coloniale française, le gouverneur général Albert LEBRUN n'avait pas manqué de prendre des initiatives adéquates à cet effet. Il déclarait dans une note circulaire: « $J^{\prime} a i$ envisagé, comme moyen efficace d'empêcher une trop grande déforestation du pays, de créer un vaste domaine forestier classé, dégagé de l'inconsistance des terres boisées vacantes et sans maîtres, bien constitué en droit, définitivement assis en superficie et spécialement protégé » (décret du 5 février 1938 portant organisation du régime forestier du territoire du Togo). De son idée sont nés entre 1939 et 1957, quatre-vingt-trois (83) sites naturels domaniaux s'étendant sur 14\% de la superficie nationale (MERF, 2001) et qui constituent l'essentiel du système national d'aires protégées du Togo. Ces aires protégées soustraites aux pressions anthropiques depuis l'époque d'Albert LEBRUN ont été depuis lors, de véritables habitats pour de nombreuses espèces constituant ainsi à l'instar des autres pays, une pierre angulaire pour la conservation de la biodiversité animale et végétale. Les troubles sociopolitiques que le Togo a connus entre 1991 et 1993 au cours de la transition démocratique ont entrainé l'envahissement de la plupart de ces aires protégées et accéléré la régression des superficies forestières (MERF, 2000). Comme corollaires on assiste entre autres à l'occupation de certaines aires protégées sur 10 à $90 \%$ de leur aire (MERF, 2014), la coupe anarchique des essences ligneuses et le braconnage.

Le Complexe d'Aires Protégées Togodo (CAPT) qui présente un grand intérêt écologique en raison de la richesse de sa biodiversité (Courcelaud, 2000) n'a pas été non plus épargné par ce fléau. Ce complexe qui est constitué du Parc National Togodo Sud (PNTS) et de la réserve de faune (Togodo Nord) est très représentatif des écosystèmes reliques de l'écorégion du couloir de Dahomey. Il renferme outre les derniers troupeaux des buffles de forêt du Togo après que la faune a été décimée lors des troubles sociopolitiques des années 90 (Adjonou, 2010), de nombreux primates non humains dont le singe à ventre rouge (Cercopithecus erythrogaster erythrogaster Gray), un petit singe frugivore dont le pelage du ventre et de la poitrine est de couleur rouge et qui mesure entre 40 et $50 \mathrm{~cm}$ pour 2 à $4 \mathrm{~kg}$. Il s'agit d'une sous-espèce en danger et endémique du Dahomey Gap (Houngbédji, 2012) longtemps considérée comme disparue de son habitat naturel jusqu'en 1987 où un spécimen vivant fut expédié depuis Lomé à Mulhouse Zoo en France. Nombre d'études ont été alors menées sur ce singe dans le Dahomey Gap depuis sa redécouverte en 1994 par Oates au Bénin. Mais jusqu'alors, seule la partie "béninoise" a fait objet d'études approfondies. Même si 
un inventaire sommaire depuis la berge béninoise a permis à Houngbédji (2010) de recenser neuf individus dans la partie Sud du complexe d'aires protégées Togodo et un autre inventaire de Campbell (2005) sur les primates non humains en général ne lui a pas permis de rencontrer la sousespèce. Face à cette insuffisance de données sur la sous espèce, il urge qu'un état des lieux soit fait à travers l'inventaire de ces sites d'occurrence aux fins de déterminer sa distribution spatiale ainsi que les différentes formes de menaces pesant sur sa survie au Togo.

\section{MATERIEL ET METHODES}

\section{Zone d'étude}

La présente étude s'est déroulée au Togo sur le complexe d'aires protégées de 25500 ha constitué de deux aires contiguës situées dans la partie togolaise du Dahomey Gap : le Parc national Togodo Sud (15 000 ha) et la Réserve de faune Togodo Nord (10500 ha) (Figure 1). Le climat est de type subéquatorial, avec deux saisons sèches alternées par deux saisons pluvieuses. On distingue une grande saison pluvieuse qui s'étend de mars à juillet et une petite saison des pluies de septembre à octobre tandis que la petite saison sèche couvre la fin du mois de juillet à septembre et la grande saison sèche de novembre à février (Figure 2). La précipitation est variable de 800 à $1200 \mathrm{~mm}$ par an et de 60 à 90 jours de pluies annuelles (Edorh-Ananou, 2005). La température moyenne annuelle est de $27{ }^{\circ} \mathrm{C}$ environ. Le complexe est constitué d'une mosaïque de forêts et de savanes caractéristiques du passage du couloir de Dahomey (Courcelaud, 2000). Les forêts de type guinéen font la transition entre la forêt semi-caducifoliée et la forêt sèche. On distingue également des mosaïques de jachère-cultures par endroits au niveau de ses périphéries. Les sols sont caractérisés par les formations de sable argileux, de sables ferrugineux et de la terre de barre très dégradée (Edorh-Ananou, 2005).

\section{Méthode d'étude}

Les approches méthodologiques qui ont permis d'identifier les sites d'occurrence du singe à ventre rouge et de les dénombrer ont consisté à alterner entre les enquêtes dans les villages et des prospections pédestres dans le complexe d'aires protégées (méthode des recces ou la marche de reconnaissance).

\section{Les enquêtes}

Elles ont été réalisées dans six villages riverains auprès des chasseurs sous forme d'entretiens personnalisés grâce aux guides d'entretien et questionnaires d'enquête. Le guide des Mammifères d'Afrique de Jonathan Kingdon (2006), celui des primates non humains de l'Afrique de l'Ouest de John F. Oates (2011) et des planches d'illustration de diverses images de primates non humains rencontrés au Togo ont été utilisés pour faciliter l'identification de l'animal. Au total 39 chasseurs ont été interviewés. Les données collectées ont porté sur la présence/absence, les habitats probables du singe à ventre rouge (la distribution géographique) et sur ses différentes formes de menaces.

\section{Les prospections pédestres}

Les prospections pédestres ont permis non seulement de dénombrer les singes à ventre rouge dans les sites probables du complexe qui ont été indiqués par les guides dans les six zones et dans la forêt sacrée de Godjinmé mais aussi d'identifier leur habitat et de recenser les éventuelles traces d'activités anthropiques. La méthode des recces encore appelée la marche de reconnaissance a été utilisée à cet effet. Il s'est agi donc, d'un recensement direct basé sur le décompte des singes à 
ventre rouge directement observés à l'œil nu ou grâce aux jumelles. Trois équipes de trois personnes (un guide, un chasseur et forestier) ont parcouru le complexe d'aires protégées pendant 4 jours par zone en empruntant les pistes des braconniers. Chaque équipe dispose d'une fiche technique qu'elle remplit au fur et à mesure que les observations se déroulent. Sont renseignées sur la fiche technique les informations suivantes : l'heure de l'observation des singes à ventre rouge, leur effectif, le type d'habitat, les coordonnées GPS des contacts (la position de l'observateur), l'estimation de la distance entre l'observateur et l'individu, l'activité au moment de l'observation. Tout indice de présence d'animaux ou d'activités anthropiques a été également enregistré sur la fiche. L'inventaire débute chaque jour à $5 \mathrm{~h} 30 \mathrm{mn}$ pour prendre fin à $14 \mathrm{~h}$ ou $15 \mathrm{~h}$ voire $17 \mathrm{~h}$. Pour éviter les doubles comptages, les équipes ont débuté l'inventaire à la même heure. Aussi, le fait que les groupes des singes se déplacent peu, permet de ne les compter qu'une seule fois. L'appréciation de l'abondance s'est limitée seulement au calcul de l'indice kilométrique d'abondance (IKA) compte tenu de la méthode utilisée. C'est le rapport du nombre d'individus observés à la distance parcourue. Sa formule se présente comme suit :

$$
\mathrm{IKA}=\frac{\mathrm{NIOE}}{\mathrm{DTP}}
$$

Avec NIOE: Nombre d'individus observés pour l'espèce ; DTP: Distance totale parcourue.

\section{Traitement des données \\ Données biophysiques}

Pour identifier et recenser les singes à ventre rouge ainsi que leurs sites d'occurrence aussi bien dans le complexe d'aires protégées que dans la forêt sacrée de Godjinmé, un système binaire de présence/absence a été utilisé. Le singe à ventre rouge est considéré comme présent :

- si les enquêtés témoignent de leur existence dans le milieu et sont à même de l'identifier clairement parmi les autres singes communs de la zone sur la planche d'illustration et de nous amener sur leur site d'occurrence ou

- si au cours des prospections pédestres dans le complexe tout comme dans la forêt de Godjinmé, un contact visuel au moins est enregistré.

Le singe à ventre à rouge est alors considéré absent s'il n'est ni signalé ni identifié par les interviewés ni rencontré lors des prospections forestières pédestres. L'inventaire des sites d'occurrence a été ainsi réalisé à partir de ces informations. La zone d'occurrence a été estimée grâce aux directives de détermination des zones d'occurrence de l'IUCN (2001). Avec les données collectées (points GPS des contacts avec la sous espèce, les traces d'activités anthropiques) au cours de l'inventaire, les sites d'occurrence ont été représentés ainsi que la répartition spatiale des singes à ventre rouge et les indices de présence humaine par l'entremise du logiciel libre Qgis 2.10.

\section{Données sur les différentes formes de menaces}

Pour mieux cerner la perception des riverains par rapport aux menaces qui pèsent sur les populations des singes à ventre rouge, des données ont été collectées au cours des entretiens par le biais des questionnaires. Les questions abordées ont porté sur la chasse des animaux en général et des primates non humains en particulier. 
Les questionnaires ont été après dépouillés et analysés sur la base des outils de statistiques descriptives du Ms Excel 2013 pour mesurer les menaces qui pèsent sur le cercopithèque à ventre rouge.

\section{RESULTATS}

Effectif des Cercopithecus erythrogaster erythrogaster, leurs zones d'occurrence et distribution spatiale dans le complexe d'aires protégées

Les observations ont été faites sur une distance de $250,14 \mathrm{~km}$ pendant environ 9 jours (213 heures). Ce qui a permis de dénombrer quatre-vingt-dix-neuf (99) individus. Etant donné qu'aucune méthode classique susceptible de nous permettre de calculer la densité absolue n'a été utilisée, seul l'indice kilométrique d'abondance (IKA) a été calculé. Il est de 0,39 individu par kilomètre. La Figure 3 donne la précision sur l'indice kilométrique d'abondance du singe à ventre rouge dans chaque secteur prospecté ainsi que celui du complexe en entier. Les résultats de l'identification des zones d'occurrence sont consignés dans le Tableau 1 qui présente la distribution du singe à ventre rouge en fonction de la préfecture, du canton et du village. L'aire d'occurrence est estimée à 93560 $\mathrm{m}^{2}$ soit $37 \%$ de la superficie totale du complexe. Elle est matérialisée par la Figure 4. La distribution spatiale des singes à ventre rouge quant à elle, est représentée dans la Figure 5.

\section{Les différentes formes de menaces recensées}

Le braconnage, la transhumance et la dégradation ou la perte de l'habitat du singe à ventre rouge constituent de sérieuses menaces pour la conservation des primates non humains en général et du singe à ventre rouge en particulier dans le complexe. En somme, on distingue deux types de menaces: les menaces directes ou le braconnage et les menaces indirectes ou la dégradation de l'habitat du singe à ventre rouge.

Le braconnage se pratique de façon illicite avec des fusils, voire avec des chiens dans le complexe d'aires protégées, par des braconniers installés dans les villages riverains. Il justifie d'ailleurs, leur proximité par rapport au complexe. Il se pratique aussi bien le jour que la nuit. Plus de $50 \%$ des interviewés reconnaissent sa pratique en tout temps et en toutes saisons (sèche et pluvieuse). Les Figures $6(\mathrm{a}, \mathrm{b})$ et 7 (c, d) illustrent la recrudescence du braconnage dans le complexe. Les singes à ventre rouge, une fois abattus ou capturés servent à l'alimentation (48,71\%), à l'usage ethno-zoologique $(5,12 \%)$ ou encore faire objet de commercialisation $(28,20)$; alors que dans $17,94 \%$ des cas selon les villageois, ils les abattent pour protéger leurs cultures (Figure 8).

Les menaces indirectes se traduisent entre autres par la transhumance, la déforestation, la dégradation et, ou la perte de l'habitat du singe à ventre rouge Figures $9(\mathrm{e}, \mathrm{f})$ et 10 . A ces menaces s'ajoute la construction du barrage hydroélectrique d'Adjarala dans l'emprise du complexe. 


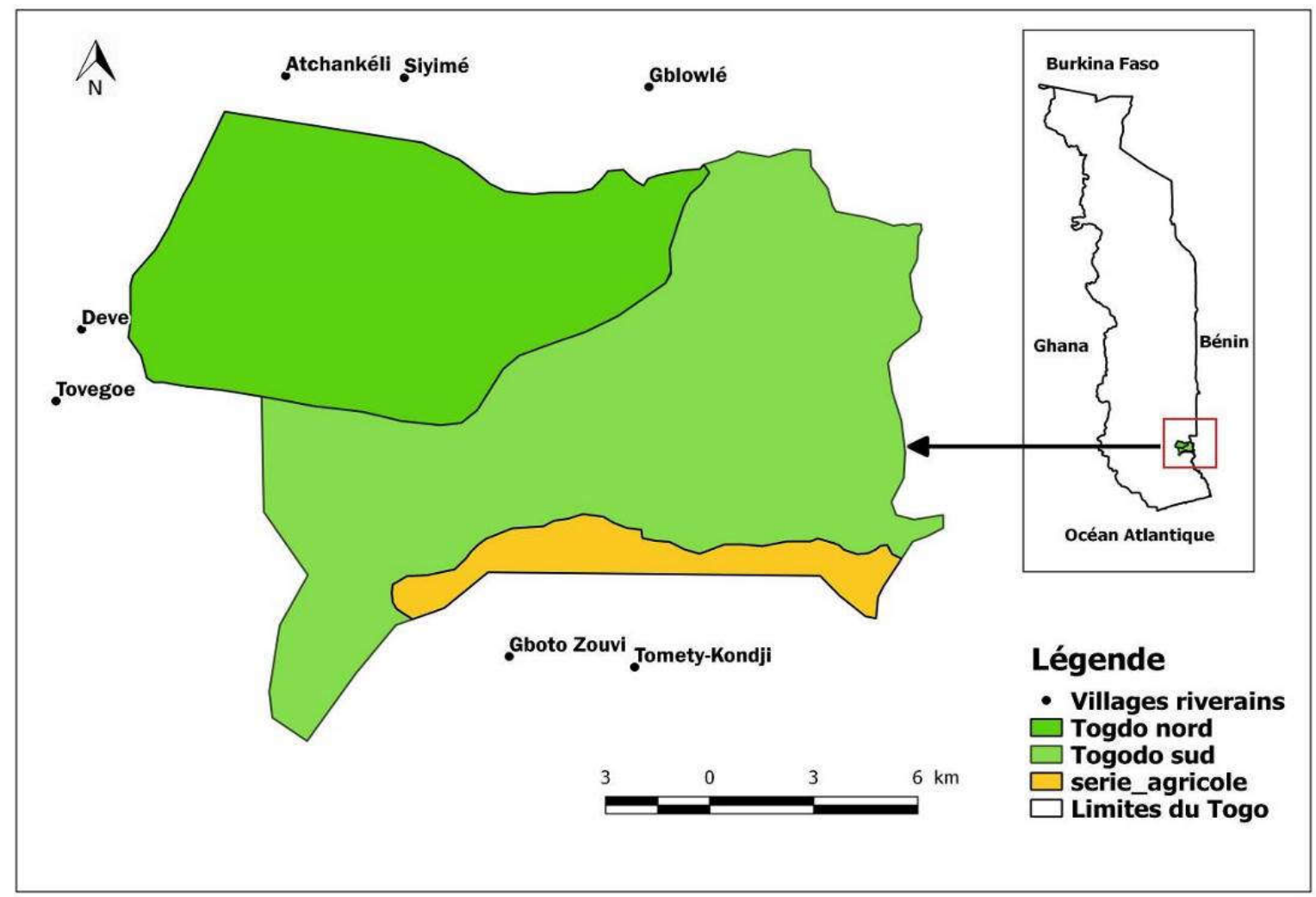

Figure 1: Localisation de la zone d'étude.

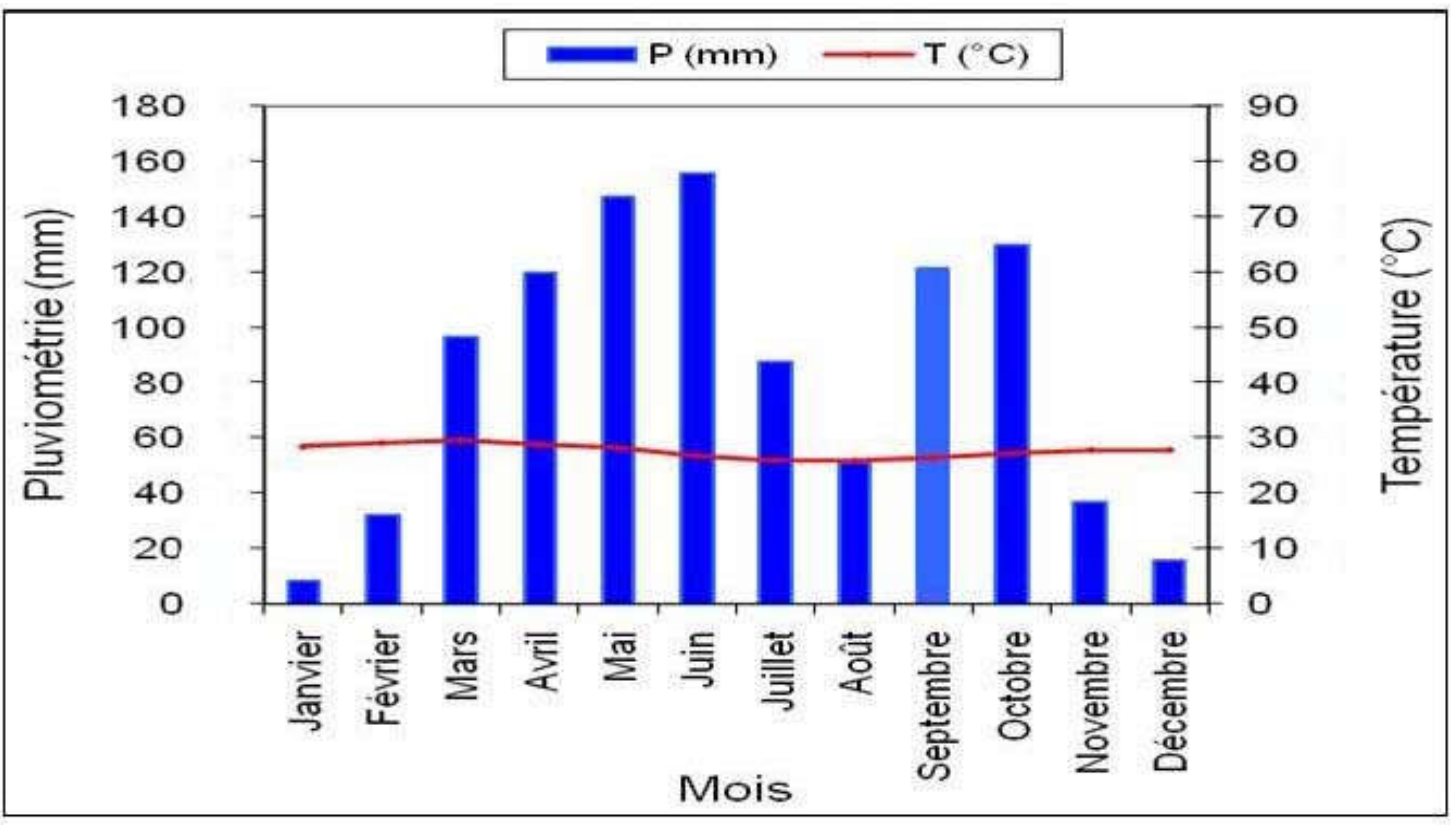

Figure 2: Diagramme ombrothermique de la station de Tabligbo (2005). (Direction Nationale de la Météorologie, 2006). 


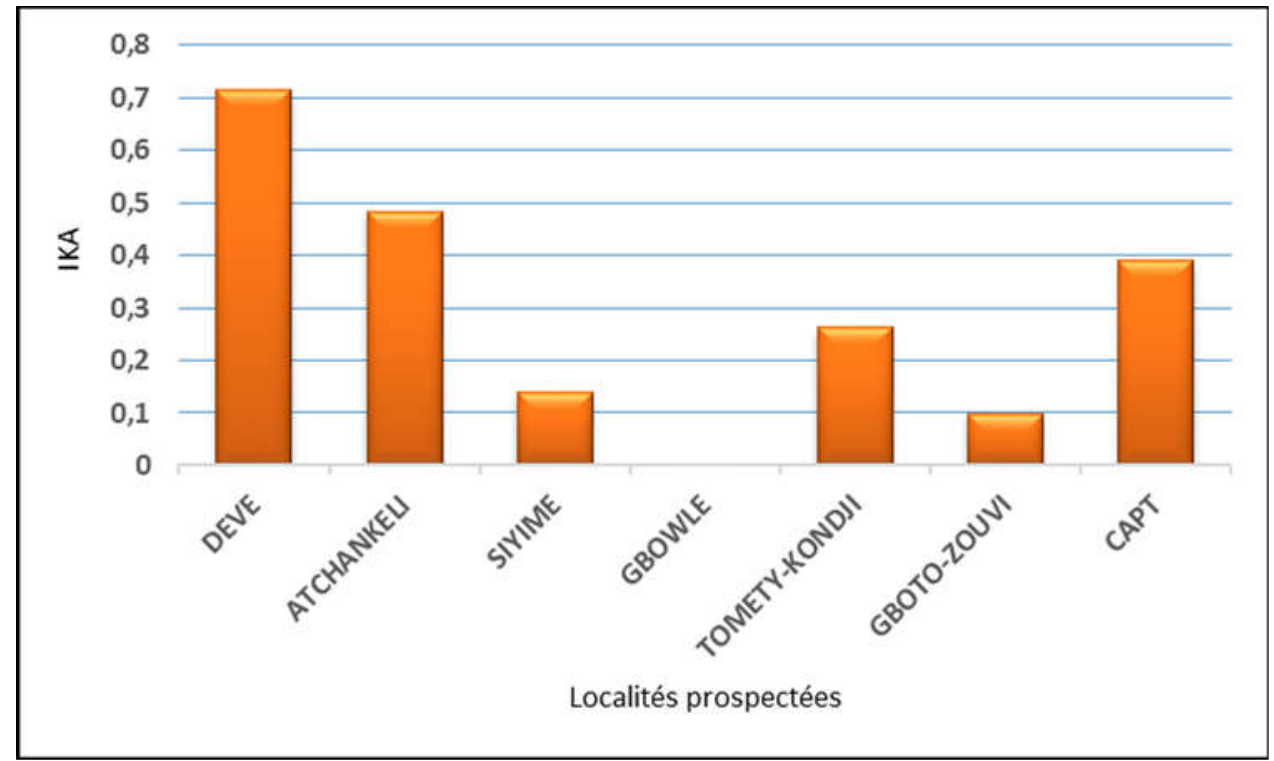

Figure 3: Indice Kilométrique d'Abondance (IKA) des différentes zones prospectées.

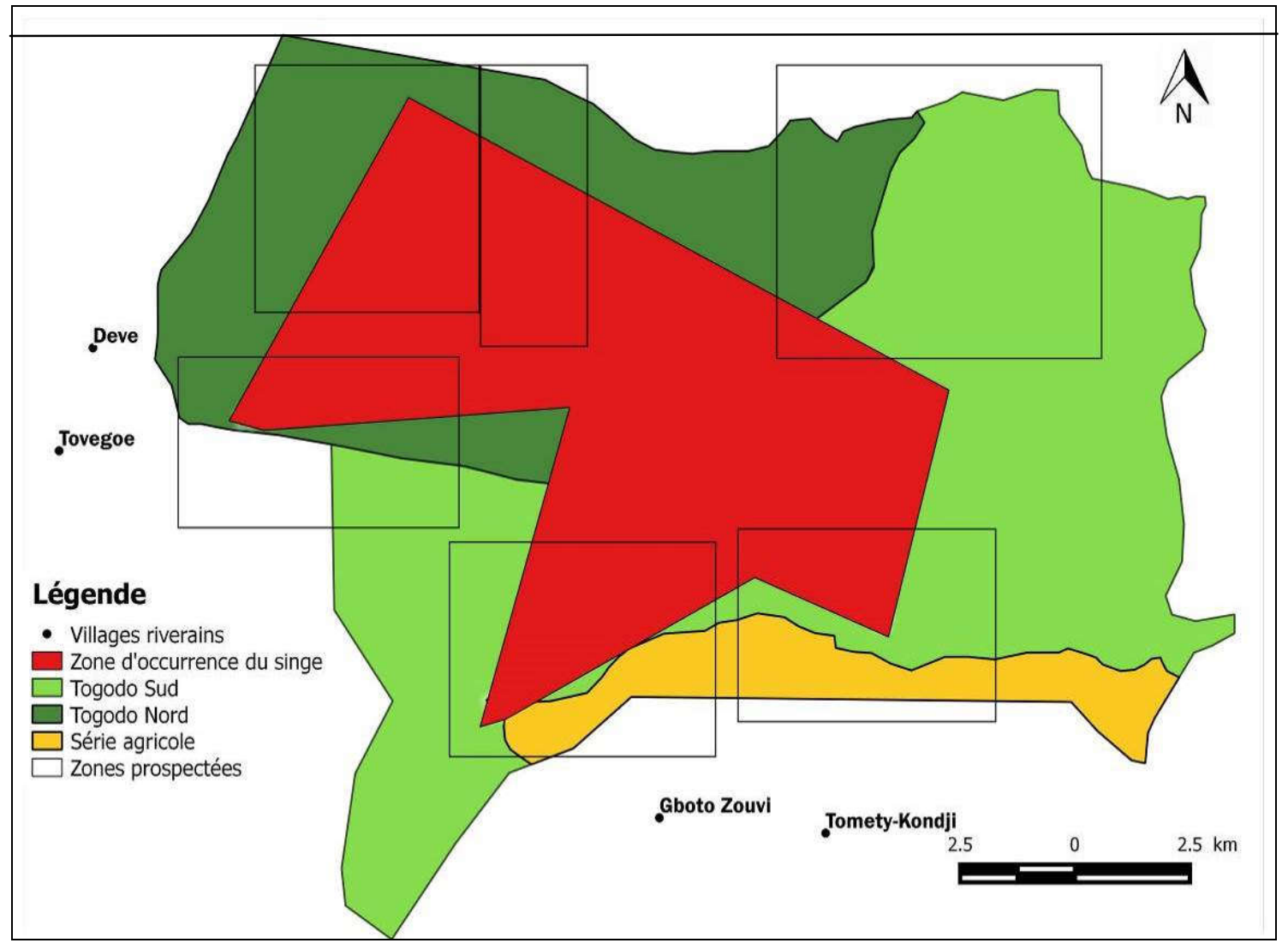

Figure 4 : Zone d'occurrence des singes au ventre rouge dans le complexe. 


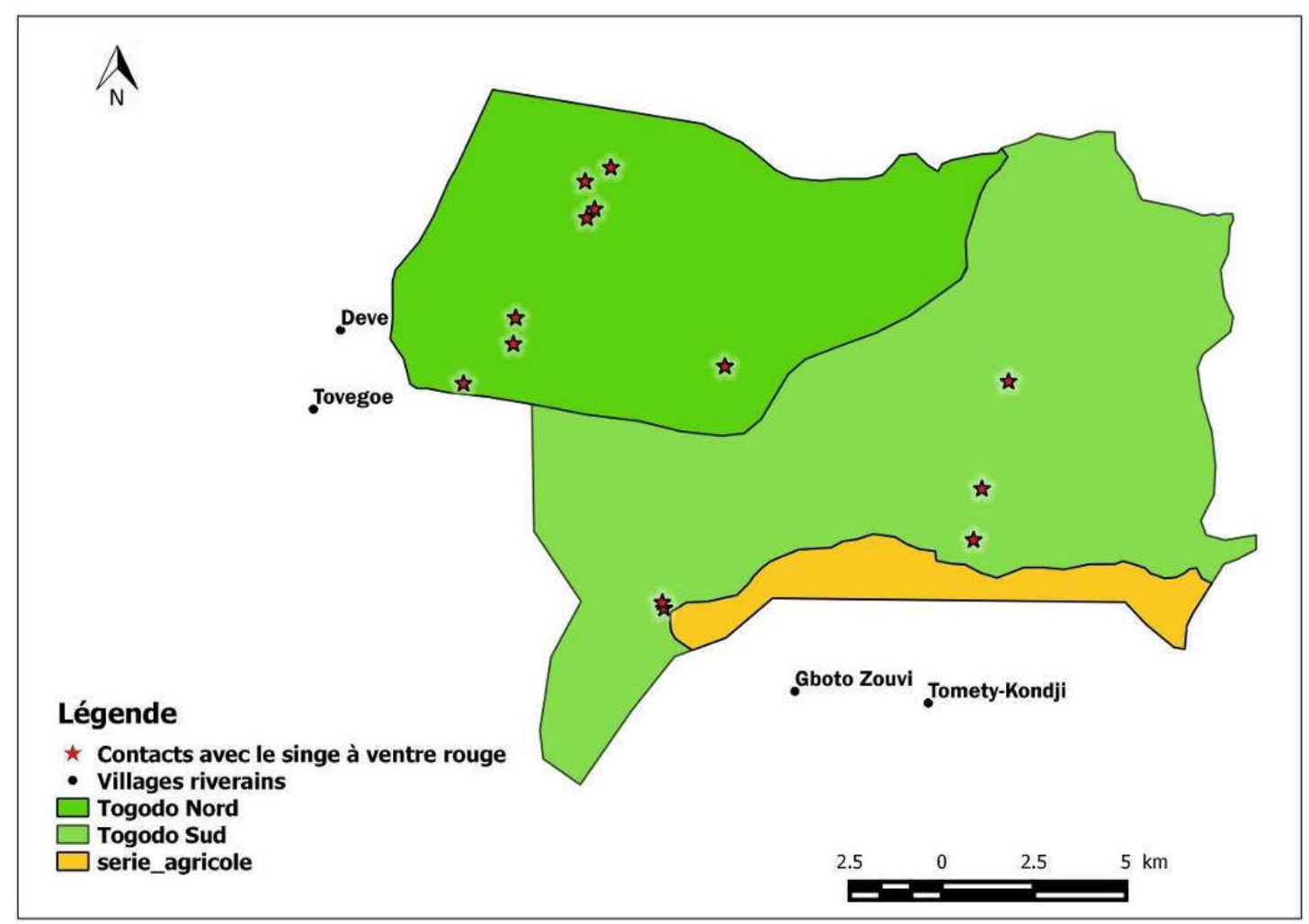

Figure 5 : Contacts avec les singes à ventre rouge.
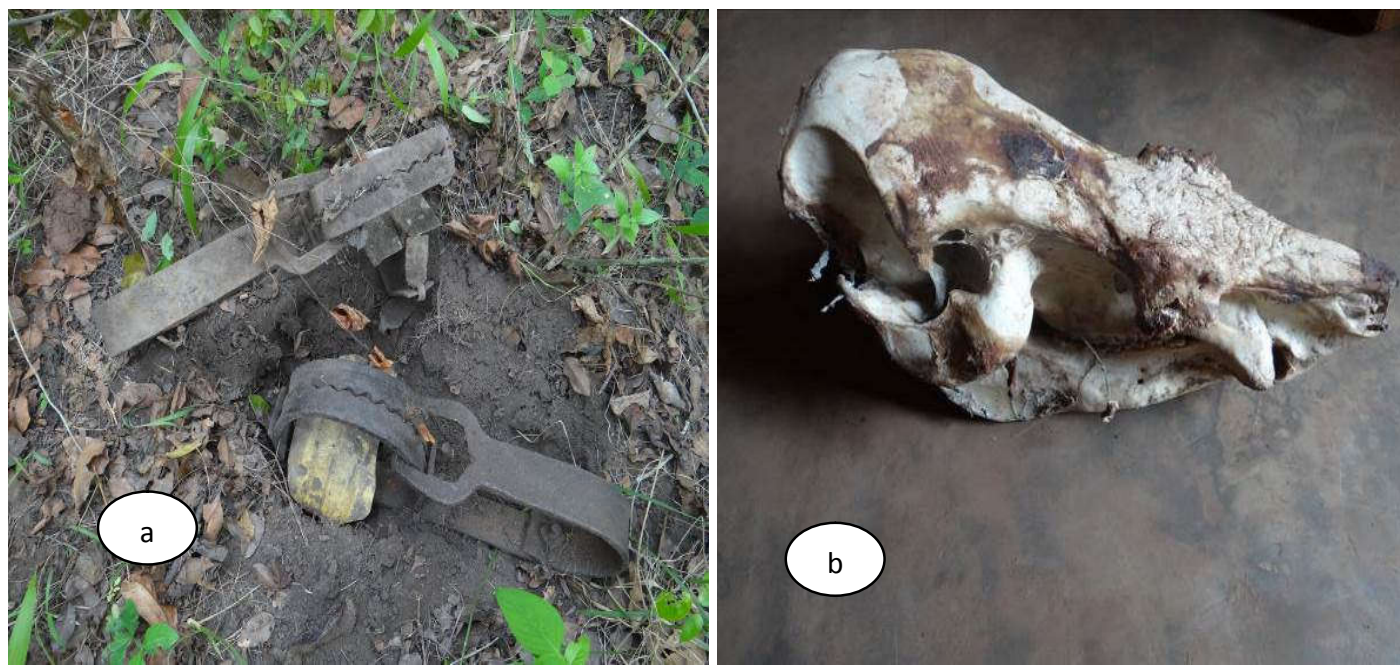

Figure 6: Pièges retrouvés dans le complexe (a) et crâne d'un potamochère chez un braconnier (b). 

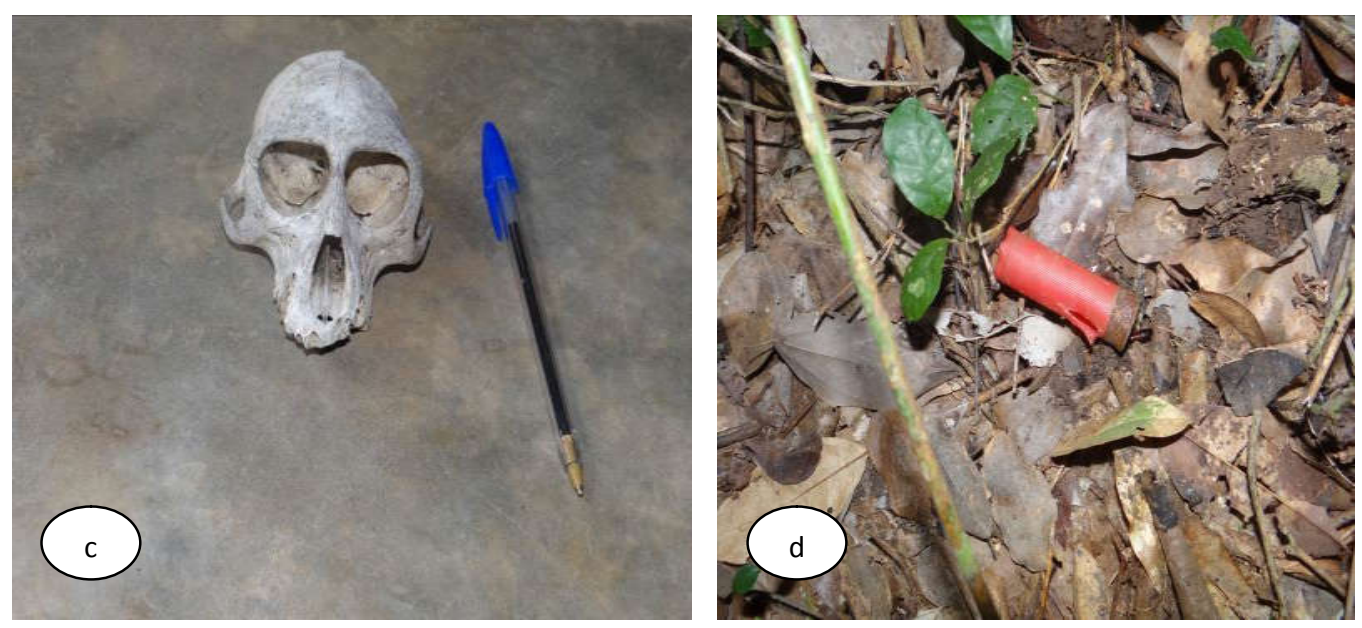

Figure 7: Douille retrouvée dans le complexe (d) et un crâne de Erythrocebus patas chez un braconnier (c).

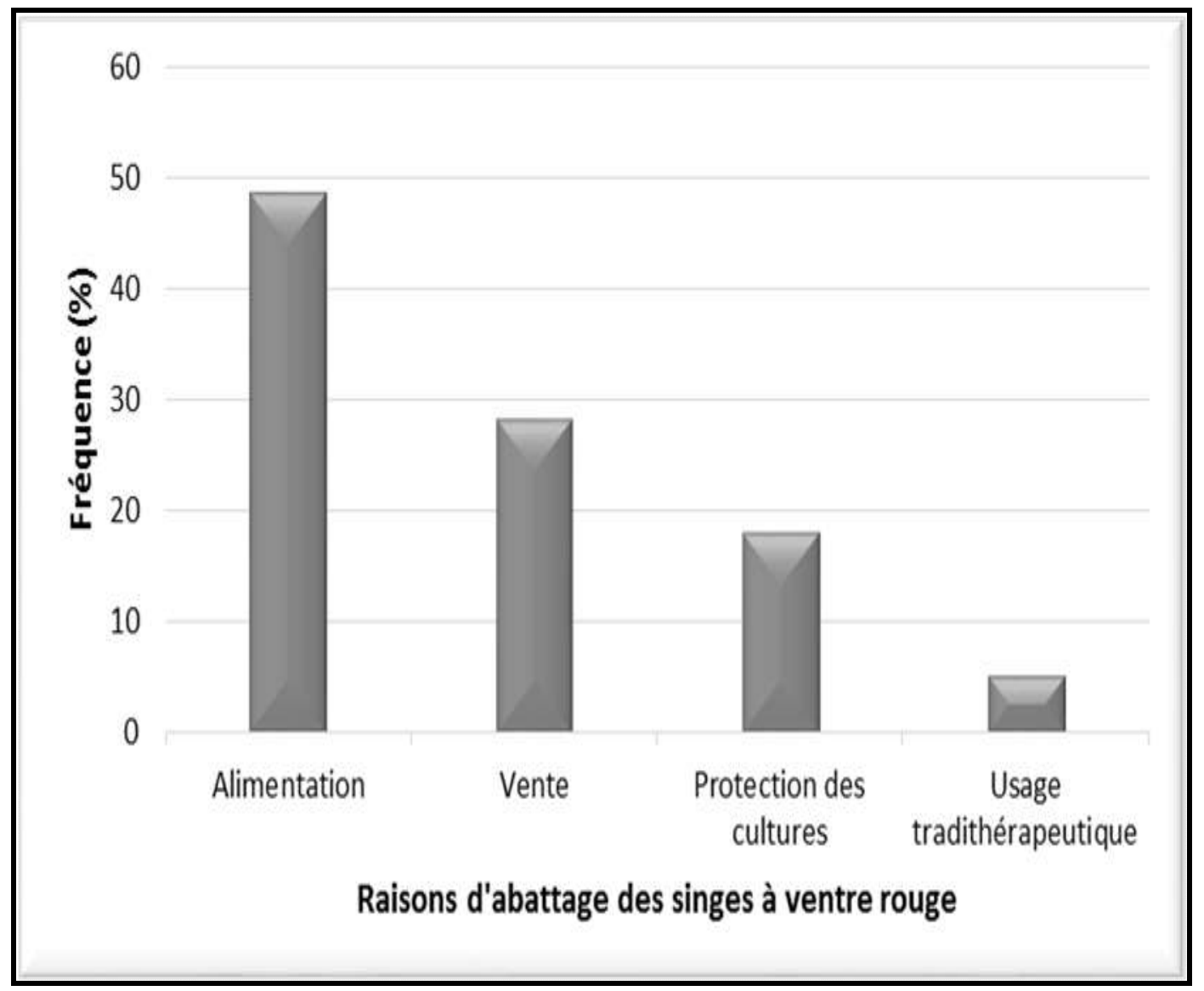

Figure 8 : Utilisation faites des singes à ventre rouge abattus. 

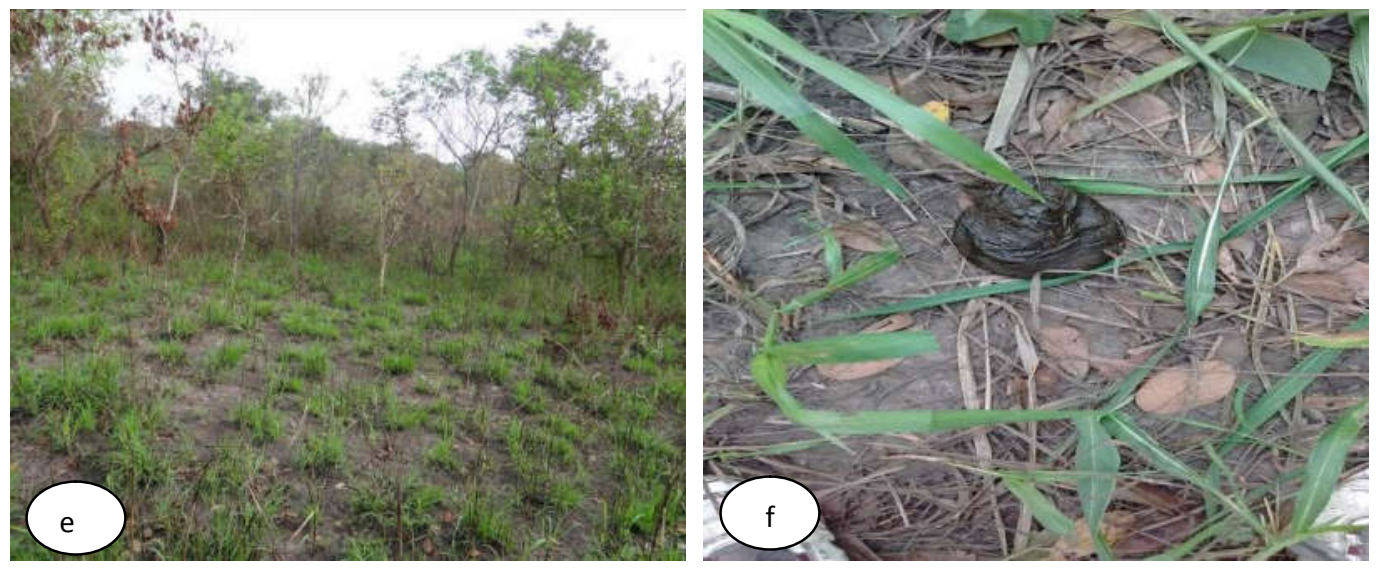

Figure 9: Déjection de bœuf (f) et l'aspect de la végétation après le passage des feux dans le complexe (zone de Dévé) (e).

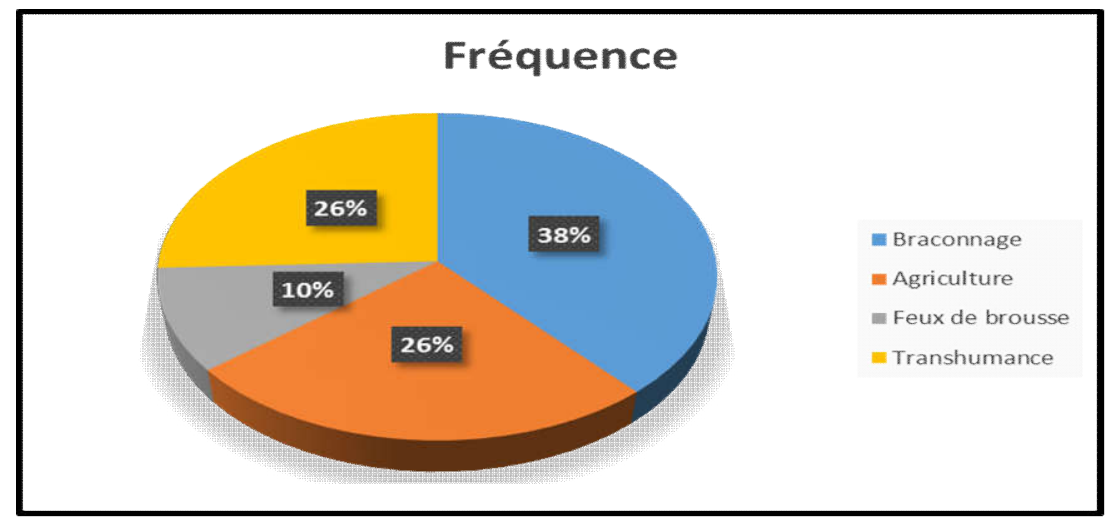

Figure 10 : L'ampleur des activités anthropiques pratiquées dans les habitats du singe à ventre rouge.

Tableau 1 : Répartition du singe à ventre rouge en fonction de la préfecture, canton, village.

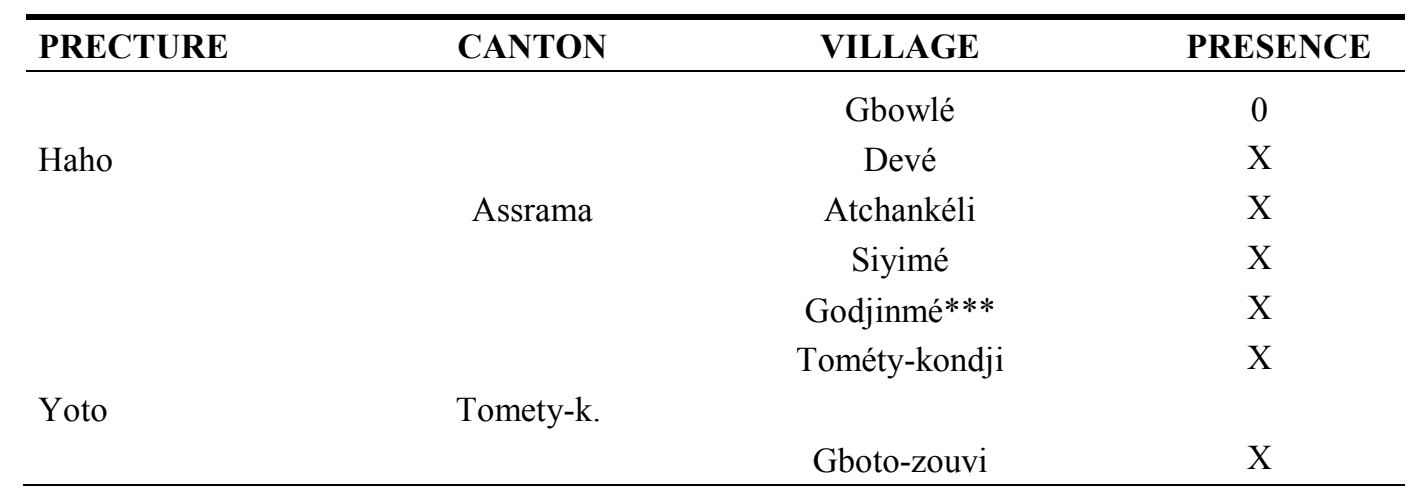

Légende : $\mathrm{O}=$ Non, $\mathrm{X}=$ Oui

*** Forêt sacrée située non loin du complexe Togodo. 


\section{DISCUSSION}

Effectif des Cercopithecus erythrogaster erythrogaster, leurs zones d'occurrence et distribution spatiale dans le complexe d'aires protégées et dans la Forêt Classée de Godjinmé

Il ressort de l'analyse des résultats d'enquêtes réalisées dans les six villages riverains (Tovégoé, Dévé, Siyimé, TométyKondji, Atchankéli et Gbowlé) du complexe d'aires protégées que le singe à ventre rouge est très bien connu par tous les 39 chasseurs interrogés. $\mathrm{Au}$ cours des prospections pédestres, les individus des singes à ventre rouge ont été essentiellement rencontrés dans les forêts semi-décidues caractérisées par des lianes qui sont leurs lieux privilégiés de jeux. On distingue également des forêts galeries (surtout de la zone de Siyimé), des sanves ou encore des forêts dégradées et des culturesjachères. La prospection dans les six villages et dans la forêt sacrée de Godjinmé a permis de réaliser des observations directes des individus de singes à ventre rouge sauf dans la zone de Gbowlé malgré l'existence de quelques forêts galeries pouvant abriter la sous espèce. Cela serait dû au fait que cette zone est fortement perturbée par le braconnage et autres activités anthropiques qui ont entraîné la fuite des primates non humains. C'est également dans cette zone que l'exploitation forestière des ligneux est la plus importante. Les essences naturelles (notamment les Pterocarpus erinaceus) sont sciées puis exploitées en bois de services et en bois d'œuvre ou transformées en pirogues pour être emportées au Bénin par le fleuve Mono d'une part, et utilisées pour la fabrication de charbon de bois d'autre part. C'est le cas de Anogeissus leiocarpus. Les résultats de quatre-vingt-dix-neuf (99) individus recensés dans le complexe et cinq (5) dans la forêt sacrée de Godjinmé ont permis de confirmer non seulement leur présence dans le complexe d'aires protégées Togodo et dans la forêt sacrée de Godjinmé, mais aussi d'identifier leur zone de distribution. En effet, dans la partie Nord du complexe où quatre-vingt-trois (83) individus ont été dénombrés, huit (8) contacts ont été enregistrés dont cinq (5) dans les forêts galerie, deux (2) dans les forêts claires et un (1) dans la savane/jachère. Quant à la partie Sud, pour seize individus (16), cinq (5) contacts ont été notés dont deux (2) dans les galeries forestières, deux (2) dans les forêts claires et un (1) dans la savane. Ceci confirme la présence régulière de cette sous espèce au Togo contrairement aux travaux de Sinsin (2002) et de Nobimè (2005) qui ne l'ont signalée que dans la partie béninoise du Dahomey Gap. Ces résultats viennent compléter les travaux de Campbell (2005) qui, malgré sa prospection dans des conditions similaires n'a pas réalisé des observations directes de la sous espèce au Togo même si elle déclare avoir entendu une vocalisation qui serait son cri. L'effectif de 99 individus recensés dans le complexe et cinq (5) dans la forêt sacrée de Godjinmé confirme en partie le résultat de 9 individus inventoriés uniquement au niveau de la partie Sud du complexe par Houngbédji (2010) mais dans des conditions un peu différentes comme la durée qui est de 72 heures seulement et, aussi la plupart des observations ont été faites depuis la berge béninoise. Cet effectif bien qu'inférieur à celui de la forêt de la Lama conforte l'assertion de Houngbédji (2015) qui estime que l'habitat source serait le complexe d'aires protégées Togodo par rapport à la dépression de Tchi (Sud du Bénin) eu égard à son étendue (plus grande que la dépression de Tchi) et au nombre d'habitats potentiels qui s'y trouvent. Sur cette base, on pourrait dire que le spécimen envoyé à Mulhouse Zoo en France par le marchand Eric Fouchard dans les années 80 serait extirpé du Sud Togo. En effet, bien avant les années 90, le Togo était cité comme un modèle en matière de protection de la faune et de son habitat. Les primates non humains faisant objet d'une conservation particulière car non seulement ils sont vénérés mais constituent une composante 
importante de la biodiversité grâce à leur rôle dans le maintien de l'écosystème forestier en contribuant largement à la dispersion des graines et à la régénération des arbres par zoochorie (Djégo-Djossou, 2009). Cependant, la gestion intégrale des aires protégées a connu ses limites avec leur envahissement par les populations riveraines révoltées pendant la période de transition démocratique entre 1991 et 1993. Ceci a contraint les animaux à fuir vers les pays voisins et vers d'autres habitats du Togo (Godjinmé par exemple). Les travaux d'Oates (1995) se sont déroulés juste après ces périodes troubles caractérisées par l'envahissement des aires protégées par les populations riveraines avec des conséquences drastiques sur la faune togolaise. C'est ainsi qu'une bonne partie de la communauté des singes à ventre rouge aurait quitté le complexe d'aires protégées et autres forêts pour se réfugier dans certaines forêts du Bénin voisin. La présence de ces singes dans la forêt sacrée de Godjinmé montre qu'ils ne sont pas confinés seulement dans le complexe mais, ont une large répartition et peuvent se retrouver dans d'autres forêts présentant des similarités avec le complexe en terme de végétation.

\section{Menaces pesant sur les singes à ventre rouge \\ Le braconnage}

Bien que toute activité de chasse sans autorisation soit prohibée dans les aires protégées du Togo par le code forestier en son article 79 , elle se pratique dans le complexe surtout pendant la nuit. Ceci constitue une grave menace pour les espèces du complexe en général et pour les singes à ventre rouge en particulier. Les animaux sont abattus sans aucune technique sélective en raison de la visibilité réduite. Ni les femelles gestantes ou suitées, ni les jeunes ne sont épargnés. Ceux qui chassent la nuit estiment qu'il s'agit d'un choix stratégique pour échapper à la vigilance des agents des eaux et forêts qui n'ont pas l'habitude de patrouiller la nuit. Aussi, pendant la chasse de nuit l'effort est-il vite récompensé et on peut se consacrer à l'agriculture pendant le jour. Pour les adeptes du jour, bien que la vigilance soit redoublée, les proies sont vite repérées et ils se sentent en sécurité sans risque d'essuyer par accident, une balle perdue d'un compère. Quant à ceux qui chassent aussi bien le jour et la nuit, il s'agit pour eux de maximiser les chances de prise de proies, car certains animaux à l'instar de la plupart des singes sont actifs très tôt le matin. La plupart des braconniers viennent des villages du Bénin voisin et n'hésitent pas à s'installer dans le complexe où ils créent des campements. Plus de $75 \%$ des chasseurs interrogés se souviennent avoir tué au moins un singe à ventre rouge ces 5 dernières années (29 individus environ) même si cela s'est passé de façon fortuite, pour la plupart des cas. Pour certains, c'est dans les pièges placés par d'autres braconniers ou cultivateurs qu'ils les retrouvent généralement. Pour les cultivateurs, les pièges sont placés pour la protection de leurs champs installés à proximité du complexe quand la technique d'épouvantail ou de sarclage le long du périmètre des champs se révèlent inefficaces pour protéger leur champ. Outre l'usage des fusils et des pièges, sont également utilisés pour la chasse, les chiens prédateurs qui facilitent l'abattage ou la capture des singes notamment. Devant les chiens suffisamment entraînés aussi bien à la course qu'aux fouilles et à la détection des proies cachées, la technique de dissimilation dans les sous-bois $\mathrm{du}$ cercopithèque à ventre rouge faiblit. Aussi, faut-il redouter la rage que ces chiens peuvent transmettre aux singes en cas de morsures. Les singes à ventre rouge, une fois abattus ou capturés servent à l'alimentation, à l'usage ethno-zoologique ou encore font objet de commercialisation. Le commerce est en effet très développé en faveur d'une forte demande de viande de brousse ou de spécimen vivant. En effet, le braconnage et la recherche de terres fertiles sont en substance, les principales raisons de l'extension des 
différents villages riverains du complexe. $38 \%$ des interviewés reconnaissent que le braconnage constitue la grande menace pour les singes à ventre rouge et que leur effectif tend à diminuer progressivement par rapport aux années antérieures. Près de $15 \%$ estiment que ce sont les peuhls transhumants qui en sont la cause. Environ $8 \%$ pensent qu'ils se déplacent peu ou restent cachés à des endroits inconnus ; ce qui fait qu'on ne les rencontre plus souvent comme auparavant. Certains ont affirmé que les différentes parties des singes interviennent dans la préparation des produits traditionnels et pratiques magiques. Ils n'ont pas voulu fournir d'autres détails. La forte pression anthropique expliquerait alors en partie, la difficulté et les longues distances parcourues parfois avant de les rencontrer dans des endroits plus ou moins calmes. La chasse non contrôlée reste un danger permanent pour la biodiversité. Ce qui est accentué par l'explosion démographique qui s'accompagne d'une augmentation de la demande en viande de brousse près des exploitations agricoles à proximité des zones de conservation (Ndengue-Mekongo, 2011).

\section{Menaces indirectes}

Elles se traduisent entre autres par la transhumance, la déforestation, la dégradation et, ou la perte de l'habitat du singe à ventre rouge et la construction d'un barrage hydroélectrique dans l'emprise du complexe.

$\checkmark$ Transhumance: La présence et le passage des troupeaux de bœufs dans le complexe à la recherche du pâturage ont un effet répulsif sur les singes qui fuient vers d'autres refuges comme la forêt sacrée de Godjinmé ou vers d'autres habitats hôtes dans le Bénin voisin. A la perte de la biodiversité par élagage, s'ajoutent le piétinement et le broutage des jeunes pousses susceptibles d'évoluer en arbres et arbustes fruitiers pouvant être consommés par les singes à ventre rouge. Le passage saisonnier des troupeaux de boufs engendre en outre, l'appauvrissement du sol par le piétinement des bœufs et le risque de propagation de maladies transmissibles.

$\checkmark$ Déforestation, dégradation et perte de l'habitat du singe à ventre rouge: presque dans tout le complexe d'aires protégées Togodo, aussi bien aux périphéries qu'à l'intérieur, on enregistre des exploitations abusives des produits forestiers ligneux et non ligneux. De nombreux exploitants pour la plupart béninois traversent le fleuve Mono qui sépare le complexe de leur territoire et y pénètrent clandestinement. Il s'ensuit alors des coupes anarchiques de bois surtout du côté de Gblowlé qu'ils font transiter par le fleuve pour les revendre au Bénin. Ce qui est constaté ces dix dernières années est, l'abattage massif de kapokier et de fromager qu'ils taillent en pirogues avant de les acheminer chez eux toujours par le fleuve Mono qui sert de limite naturelle entre les deux pays. Certains exploitants venant notamment des villages et agglomérations riverains comme Kouvé déterrent les racines de certaines lianes susceptibles de servir d'habitat pour les singes à ventre rouge dont on dit avoir une vertu aphrodisiaque. Dans certains villages comme Gboto-Zouvi, les champs sont contigus au complexe. La zone tampon n'est pas respectée. De nombreux pesticides et engrais divers sont utilisés par les agriculteurs dans ces champs dont les dérivés se retrouvent dans les cours d'eau qui traversent le complexe. Tout ceci échappe à la vigilance des agents des eaux forêts et constitue de graves dangers pour les hocheurs à ventre rouge qui fréquentent régulièrement les cours d'eau et les forêts galeries. En effet, bien qu'elle soit une aire protégée (catégorie II), le complexe n'est pas géré de façon efficace. Le gestionnaire ou conservateur est généralement un technicien supérieur des eaux et forêts formé en trois ans après le baccalauréat dans un institut agricole du pays. Il ne dispose pas d'autres formations en matière de gestion des aires protégées. Il est assisté seulement de quatre agents pour la surveillance de la partie Sud du complexe de 
15000 ha. Il en est de même pour la partie Nord de 10500 ha. Leurs seuls moyens se résument à leurs vieilles armes "Mas 36" et leur force physique pour les patrouilles. Ils ne disposent ni de budget ni de plan de gestion et de planning d'activités. Les patrouilles s'organisent sur informations avec les moyens de bord et ne couvrent pas tout le complexe. L'effectif très réduit du personnel en est la raison. Il n'existe pas de pistes aménagées pour les patrouilles. Celles utilisées sont celles des braconniers qui les maîtrisent mieux que les agents de patrouilles. Il a été rapporté que les agents sont parfois victimes de trafic d'influence de la part des militaires (notamment des officiers supérieurs des FAT) qui chassent sans permis. C'est dire que les moyens investis pour la protection du complexe sont largement en dessous des attentes. Ceci encourage les braconniers qui vont jusqu'à soudoyer les agents selon certaines indiscrétions. Aussi, il semblerait que les agents perçoivent leur affectation dans les aires protégées comme une sanction disciplinaire à leur égard.

De nombreux foyers de carbonisation ainsi que l'agriculture extensive en réponse aux besoins d'une population sans cesse croissante sont en substance les principales causes de la déforestation et des facteurs qui contribuent à la dégradation et à la perturbation de l'habitat du singe à ventre rouge. Face au manque de ressources dû à ces activités anthropiques, les populations de singes à ventre rouge qui sont des singes de forêt sont obligés de s'alimenter dans les champs installés dans les zones périphériques du complexe se laissant ainsi découvrir par les braconniers ou se retrouvent emprisonnés dans les pièges dressés par les cultivateurs. Tout ceci accentue non seulement la fragmentation et l'isolation mais aussi la vulnérabilité des populations de la sous espèce.

$\checkmark \quad$ Installation des ruches dans les zones tampons du complexe: dans certaines zones tampons comme c'est le cas à Gboto-Zouvi, sont installées des ruches menaçant ainsi l'intégrité du complexe et favorisant le passage et l'entrée des braconniers dans le complexe. Cette promiscuité incite également d'autres activités anthropiques dans le complexe. En effet, les zones tampons qui sont des espaces séparant les aires protégées des espaces exploitables et exploitées par les villages riverains sont non seulement empiétées par endroits par l'agriculture mais aussi exploitées officiellement pour l'apiculture initiée par l'ONG de développement au nom d'Amis De la Terre (ADT). Les ruches sont ainsi disposées pèle mêle le long des zones tampons engendrant des présences permanentes des riverains et des incursions dans le complexe. Ces zones tampons ne sont pas suffisamment marquées et matérialisées. Or le but des zones tampons est de créer une zone peu attrayante entre la zone de conservation et les cultures alentours. Cela implique le débroussaillage de la forêt secondaire à la limite et l'établissement d'une zone d'une certaine largeur entre la limite de la zone de conservation et les cultures (Triplet, 2009). Il s'agit là encore une fois d'une porte officielle ouverte aux braconniers et aux exploitants forestiers.

$\checkmark$ Construction du barrage hydroélectrique d'Adjarala : La construction du barrage hydroélectrique d'Adjarala est un projet commun entre le Togo et le Bénin pour juguler le déficit en énergie électrique auquel les deux pays font face. Cependant il empiète sur une bonne partie du complexe et, une analyse poussée de l'emprise du lac de son barrage montre des pertes importantes en terres dont 9100 ha de terres agricoles, 3335 ha de savanes et 560 ha d'îlots forestiers (Adjonou, 2010). Au-delà de la régression des superficies forestières et de la perte de la biodiversité, sont à craindre la fragmentation, la perturbation ou la disparition de l'habitat des cercopithèques à ventre rouge, et l'envahissement des populations riveraines du reste du complexe ainsi que la recrudescence $\mathrm{du}$ braconnage qui sera favorisée par 
l'ouverture des pistes facilitant l'accès au complexe. En outre, l'ouvrage peut constituer un obstacle physique à leur déplacement. Ils peuvent s'y retrouver piégés facilitant ainsi leur capture ou leur abattage par les braconniers. Nombre de zones prospectées où les singes à ventre rouge ont été observés sont également affectées. Ce qui présage la disparition de cette sous espèce au Togo si aucune précaution n'est prise.

\section{Conclusion}

La présente étude a permis de mieux connaître la distribution spatiale et l'état de conservation des populations des singes à ventre rouge dans le complexe d'aires protégées Togodo. Les résultats concluants obtenus dans les sites d'occurrence prospectés permettent de confirmer la présence de singes à ventre rouge non seulement dans le complexe d'aires protégées Togodo (99 individus dénombrés) mais aussi dans une autre forêt refuge: la forêt sacrée de Godjinmé (5 individus recensés). Ceci prouve que les cercopithèques à ventre rouge sont endémiques du Dahomey Gap, au sud Togo et au Bénin et non au niveau du Bénin seulement comme beaucoup de chercheurs le pensaient. Malheureusement ils sont à l'instar des autres espèces, menacés par le braconnage et la destruction à un rythme effrayant de leur habitat en raison d'une croissance démographique galopante. Il urge alors qu'un certain nombre d'actions de protection et de sauvegarde soient menées par tous les acteurs de la conservation afin de le sauver de l'extinction.

\section{INTERETS CONCURRENTS}

Les auteurs déclarent qu'ils n'ont aucun conflit d'intérêts.

\section{CONTRIBUTIONS DES AUTEURS}

Les contributions des auteurs ont été diverses et riches. KGEA est le principal investigateur; le manuscrit a été entièrement rédigé par lui et est extrait de son mémoire de Master. MO a été le Maître de stage et a suivi le travail de bout en bout depuis le protocole de recherche jusqu'à la rédaction du mémoire final. Le projet d'article lui avait été soumis en premier et il n'a pas manqué d'apporter son amélioration et ses conseils pour l'amélioration globale de l'article. MC a eu à contribuer énormément à la rédaction de cet article qui fut en outre le premier auteur, l'un des instigateurs pour avoir été le premier codirecteur de notre mémoire de Master d'où est extrait l'article. Il a procédé à la plupart des corrections avec sa rigueur scientifique et surtout ses orientations on ne peut plus pertinentes qui ont permis d'améliorer substantiellement la qualité du document final et du présent article. Il a été très disponible à apporter de son expérience pour la rédaction de l'article. HS : il a été le $2^{\mathrm{e}}$ co-directeur de notre document source d'où est née l'idée de rédaction de l'article. Les données ont été collectées sur le terrain avec son équipe de recherche. Il nous a fourni également toute la logistique nécessaire pour cette collecte sur le terrain. Nous avons énormément bénéficié de son expérience aussi bien du terrain qu'en matière de rédaction scientifique. Il nous a fait des suggestions pertinentes qui ont apporté une plus-value non moins importante à la qualité de notre article. MBH: la grande partie de la documentation consultée nous a été fournie par lui pour avoir mené des recherches similaires dans son pays le Bénin. Depuis le protocole de recherche jusqu'à la rédaction finale du mémoire et de l'article, il a été sollicité pour ses observations. Ses pertinentes orientations ont été prises en compte dans l'article tout comme dans le document du mémoire. ATK a supervisé tout le travail du mémoire et de l'article. Il fait partie de ceux qui ont jugé utile de publier les résultats du mémoire dans un article et nous a suggéré votre journal. Il a eu à nous donner les grandes orientations de l'article en passant par l'amélioration du protocole de recherches dès le tout début de la recherche. 


\section{REFERENCES}

Adjonou K. 2011. Structure et indicateurs biologiques de gestion durable des reliques de forêts sèches au Togo. Thèse de doctorat, Université de Lomé, Togo, p. 170.

Adjonou K, Kokutse AD, Kokou K. 2013. Dynamique spatiale et diversité floristique de la Réserve de Faune de Togodo au Sud Est du Togo (Afrique de l'Ouest). Scripta Botanica Belgica, 50: 63-72.

DOI: www.br.fgov.be/RESEARCH/EDITION /SBB

Afélu B, Kokou K. 2015. Paramètres physiques d'évaluation du comportement des feux de végétation au Togo. International Journal of Biological and Chemical Sciences, 9(4): 2091-2105. DOI: http://ajol.info/index.php/ijbcs

Campbell G. 2005. Distribution, Census and Habitat Preferences of Primate Species in the Dahomey Gap (West Africa), with Particular Emphasis on the Red-bellied Guenon (Cercopithecus erythrogaster erythrogaster). Master of Arts/University of Calgary, Benin City, p. 114.

Campbell G. 2007. Distribution of Diurnal Primate Species in Togo and Bénin. Folia Primatologica, 79:15-30. DOI : www.karger.com/Journal/Issue/233752

Courcelaud A. 2000. Ressources forestières de la Réserve de faune de Togodo (Sud Togo) : dynamique et diagnostic d'une gestion participative. Mémoire d'Ingénieur des Eaux et Forêts, Engref, Montpellier, France, p. 148.

Djego-Djossou S, Sinsin B. 2009. Distribution et statut de conservation du colobe de Geoffroy (Colobus vellerosus) au Bénin. International Journal of Biological and Chemical Sciences, 3(6): 1386-1397. DOI: http://indexmedicus.afro.who.int

Edorh-Ananou MSB. 2005. Reconstitution du milieu par un développement intégré autofinançant avec des systèmes économiques aux micros crédits sans intérêt pour la prévention et la protection de l'environnement. Projet de développement socio-économique de la préfecture de Yoto, $10 \mathrm{p}$.

Houngbédji BM. 2010. Etat de conservation $\mathrm{du}$ singe à ventre rouge (Cercopithecus erythrogaster erythrogaster) dans la dépression de Tchi au Sud-Benin. DESS en Aménagement et Gestion des Ressources Naturelles, Université d'Abomey-Calavi, Cotonou, p. 93.

Hougnédji BM, Djossa BA, Adomou AC, Dakpogan C, Sinsin B, Mensah GA. 2012. Conservation Status of the Redbellied Guenon (Cercopithecus erythrogaster erythrogaster) in the West Dahomey Gap in Southwestern Benin and the Adjacent Togodo Forest Reserve, South Togo. African Primates, 7(2): 184-192. DOI: http://www.academia.edu/3836024/Cons ervation_Status_of_the_Red-

bellied_Guenon_Cercopithecus_erythrog aster_erythrogaster

Kingdon J. 1997. The Kingdon Field Guide to African Mammals. Academic Press: San Diego.

Kingdon J. 2004. Guide des Mammifères d'Afrique. A\&C Black Publishers Ltd: England.

Kokou K, Adjossou K, Hamberger K. 2005. Les forêts sacrées de l'aire Ouatchi au sud-est du Togo et les contraintes actuelles des modes de gestion locale des ressources forestières. Vertigo, 6(3): 125-131. DOI : https://vertigo.revues.org/2456

MERF (Ministère de l'Environnement et des Ressources Forestières). 2008. Loi $\mathrm{n}^{\circ}$ 2008-005 portant loi-cadre sur l'environnement au Togo. JO du 08 mai 2008.

Ministère de l'Environnement et des Ressources Forestières, 2008. Loi $\mathrm{n}^{\circ}$ 2008-009 portant loi-cadre sur l'environnement au Togo. JO du 06 juin 2008. 
Ministère de l'Environnement et des Ressources Forestières, 2014. Stratégie et Plan d'Action National pour la Biodiversité du Togo. SPANB 20112020.

Ndengue Mekongo L.S. 2011. Evaluation du statut de conservation des primates diurnes à la périphérie nord-est du parc national de Korup, sud-ouest Cameroun. Mémoire de Master Université de Dschang, Caméroun, p.75.

Nobimè G, Gaoue OG, Sinsin B. 2008. Distribution des espèces de primates au Bénin et ethnozoologie. International Journal of Biological and Chemical Sciences 2(3): 346-354. DOI: http://www.ajol.info

Nobime G, Sinsin B, Lernould J-M. 2009. Ecological factors determining the distribution of the red-bellied guenon Cercopithecus e. erythrogaster in Benin and Togo. International Journal
Biological Chemical Sciences 3(3): 606611. DOI: http://www.ajol.info

Oates FJ. 2011. Primates of West Africa. A Field Guide and Natural History. Conservation International Tropical Field Guide Series: USA.

Sinsin, B, Nobimè G, Tèhou AC, Bekhuis $P$, Tchibozo S. 2002. Past and present distribution of the red-bellied guenon (Cercopithecus erythrogaster erythrogaster) in Benin. Folia Primatologica, 73: 116-123. DOI: www.karger.com/Journal/Issue/228582

Triplet P. 2009. Manuel de gestion des aires protégées d'Afrique francophone. Awely : Paris.

UICN/PACO. 2008. Evaluation de l'efficacité de la gestion des aires protégées: aires protégées du Togo. UICN-PACO: Ouagadougou. 